LU TP $98-18$

September 1998

\title{
Production mechanisms of charm hadrons in the string model
}

\author{
E. Norrbin' and T. Sjöstrand $\{$ \\ Department of Theoretical Physics, \\ Lund University, Lund, Sweden
}

\begin{abstract}
In the hadroproduction of charm in the context of string fragmentation, the pull of a beam remnant at the other end of a string may give a charm hadron more energy than the perturbatively produced charm quark. The collapse of a low-mass string to a single hadron is the extreme case in this direction, and gives rise to asymmetries between charm and anticharm hadron spectra. We study these phenomena, and develop models that describe the characteristics not only of the charm hadrons but also of the associated event.
\end{abstract}

${ }^{1}$ emanuel@thep.lu.se

${ }^{2}$ torbjorn@thep.lu.se 
Asymmetries in the production spectra of charm and anticharm hadrons (or generally, asymmetries between leading and non-leading particles) have been observed since long [1]. But it is only in recent years [2, 3, 国 that the precision has improved so as to allow more detailed studies, especially in $\pi^{-} \mathrm{p}$ events. Perturbative QCD calculations predict only very small asymmetries [5, 6], so the origin of the observed asymmetries has to be sought in nonperturbative physics.

Furthermore, the observed longitudinal momentum spectra of charm mesons are about as hard as or, in some cases, even harder than the perturbatively calculated charm quark spectra. This runs counter to naive expectations, e.g. based on experience from $\mathrm{e}^{+} \mathrm{e}^{-}$ annihilation experiments in the $10 \mathrm{GeV}$ region, where the charm hadrons only keep about two thirds of the original charm quark energy [7]. This number ought to be relevant for the hadroproduced charm events, since the $Q^{2}$ scales involved are comparable - by QCD scaling violations, the fraction slowly drops with energy.

Several scenarios have also been proposed to explain the phenomenology [8, 9, 10]. The two most frequently used ones are probably those of string fragmentation [9] and intrinsic charm [10]. In this letter we develop and study the string fragmentation approach. The key objective is to study the collapse of low-mass strings into a single hadron, which provides the main mechanism of flavour asymmetries in our approach. The outline of the letter is as follows. First we classify the different production channels for D mesons in hadron-hadron collisions, from perturbative and nonperturbative perspectives. Then we identify the origin of the charm asymmetry and compare the current model with data. The critical aspects of charm production are thereafter studied in more detail and different model variations are proposed to better understand the data. Further details and applications are intended to appear in a future paper.

The production of a charm hadron can be subdivided into two steps: first the production of a c $\bar{c}$ pair, followed by the hadronization of these quarks. We will assume that the first step of this process is adequately described by standard perturbation theory and conventional parton distributions, i.e. without the inclusion of any intrinsic charm component in the proton wave function. The charm sea is thus assumed perturbatively calculable and peaked at small $x$ values. Intrinsic charm and other modifications to standard perturbative results may well exist at some level, but here we want to show that it is possible to understand existing data without invoking new mechanisms for the production stage.

Also with this restriction, it is not possible to obtain unambiguous perturbative predictions: results are sensitive to ill-determined parameters such as the charm mass. As a simplification, we will stay with lowest-order matrix elements for charm production, augmented by a parton-shower approximation to higher-order corrections. Given the uncertainties already noted, this appears adequate.

The interesting phenomenology thus appears in the hadronization stage of our model. Here the partons of the hard interaction and of the beam remnants are connected by strings, representing the confining colour field [11. Each string contains a colour triplet endpoint, a number (possibly zero) of intermediate gluons and a colour antitriplet end. The string topology can usually be derived from the colour flow of the hard process. For instance, consider the process $u \bar{u} \rightarrow c \bar{c}$ in a $\pi^{-} p$ collision. The colour of the incoming $u$ is inherited by the outgoing $\mathrm{c}$, so it will form a colour-singlet together with the proton remnant, here represented by a colour antitriplet ud diquark. In total, the event will thus contain two strings, one $\mathrm{c}-\mathrm{ud}$ and one $\overline{\mathrm{c}}-\mathrm{d}$ (Fig. [1a). In $\mathrm{gg} \rightarrow \mathrm{c} \overline{\mathrm{c}}$ a similar inspection shows that two distinct colour topologies are possible. Representing the proton remnant 


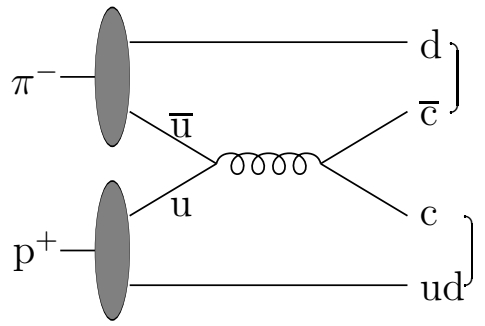

(a)

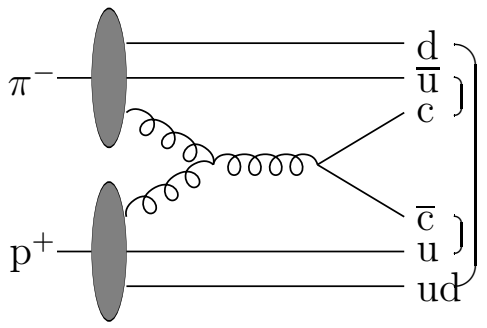

(b)

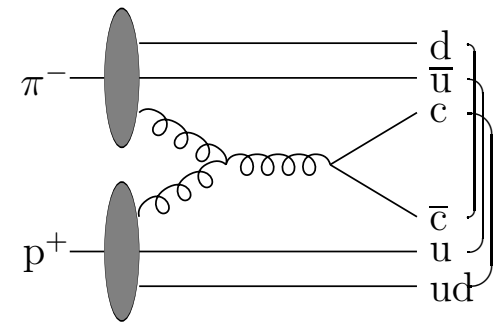

(c)

Figure 1: Examples of different string configurations in a $\pi^{-} \mathrm{p}$ collision: $(\mathrm{a}) \mathrm{u} \overline{\mathrm{u}} \rightarrow \mathrm{c} \overline{\mathrm{c}}$ has a unique colour flow; $(\mathrm{b}, \mathrm{c}) \mathrm{gg} \rightarrow \mathrm{c} \overline{\mathrm{c}}$ with the two possible colour flows.

by a $\mathrm{u}$ quark and a ud diquark (alternatively d plus uu), one possibility is to have three strings $\mathrm{c}-\overline{\mathrm{u}}, \overline{\mathrm{c}}-\mathrm{u}$ and $\mathrm{d}-\mathrm{ud}$ (Fig. 面b), and the other is the three strings $\mathrm{c}-\mathrm{ud}, \overline{\mathrm{c}}-\mathrm{d}$ and $\mathrm{u}-\overline{\mathrm{u}}$ (Fig. 11c). In addition to the valence $u \bar{u}$ annihilation and gg fusion production mechanisms, there are of course other possibilities, for example events involving sea quarks. These are included in the model but give small contributions to asymmetries and other observables at fixed-target energies, and therefore will not be discussed specifically in the following.

In a process with two (or more) allowed colour flows, such as gg $\rightarrow c \bar{c}$, the amplitude for either of them is calculable in perturbation theory, but with a nonvanishing interference term between the two. This term, corresponding to an indeterminate colour flow, fortunately is suppressed by a colour factor $1 / N_{\mathrm{C}}^{2}=1 / 9$. Its contribution to the total charm cross section therefore can be split between the two well-determined colour flows, e.g. according to the pole structure of the terms [12], without much resulting ambiguity. Furthermore, we neglect the possibility of the c $\overline{\mathrm{c}}$ pair forming a string, either by a perturbative or nonperturbative colour (re)arrangement. Such mechanisms are likely to play a significant rôle for $\mathrm{J} / \psi$ production, e.g., but should be less relevant for the open charm production to be considered here.

Once the string topology has been determined, the Lund string fragmentation model 111 can be applied to describe the nonperturbative hadronization. Assuming that the fragmentation mechanism is universal, i.e. process-independent, the good description of $\mathrm{e}^{+} \mathrm{e}^{-}$annihilation data should carry over. The main difference between $\mathrm{e}^{+} \mathrm{e}^{-}$and hadronhadron events is that the latter contain beam remnants which are colour-connected with the hard-scattering partons. The structure of these remnants is not calculable from first principles, so this introduces some arbitrariness not constrained by $\mathrm{e}^{+} \mathrm{e}^{-}$data. In the present model these aspects are parameterized in beam remnant distribution functions to be considered later.

Depending on the invariant mass of a string, practical considerations lead to the need to distinguish three hadronization prescriptions:

1. Normal string fragmentation. In $\mathrm{e}^{+} \mathrm{e}^{-}$collisions the string system has a mass equal to the full CM energy, neglecting the not-too-frequent $\mathrm{g} \rightarrow \mathrm{q} \overline{\mathrm{q}}$ shower branchings which splits a string in two. This situation is ideal for an iterative fragmentation scheme, for which the assumption of a continuum of phase-space states is essential. The average multiplicity increases linearly with the string 'length' which means, neglecting gluon-emission effects, logarithmically with the string mass. In practice, this approach can be used for all strings above some cut-off mass of a few GeV.

2. Cluster decay. In a hadron collision at fixed-target energies it frequently happens that a colour-singlet system contains two partons moving in the same general di- 


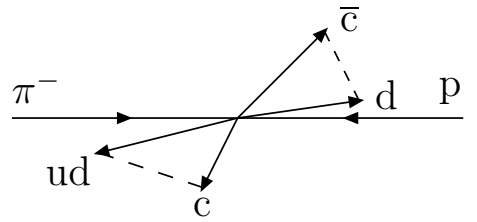

(a)

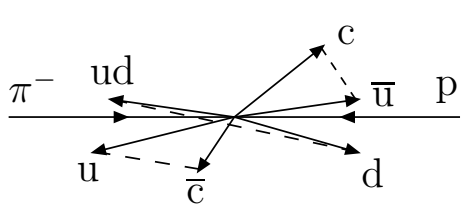

(b)

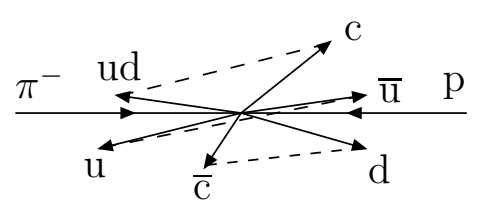

(c)

Figure 2: Strings (dashed) in a $\pi^{-} \mathrm{p}$ collision corresponding to the colour flows in Fig. 1- $1 \mathrm{a}$, $\mathrm{b}$ and c respectively; (a) $\mathrm{u} \overline{\mathrm{u}} \rightarrow \mathrm{c} \overline{\mathrm{c}}$ and $(\mathrm{b}, \mathrm{c}) \mathrm{gg} \rightarrow \mathrm{c} \overline{\mathrm{c}}$. If e.g. the colour-singlet system $\mathrm{c}-\overline{\mathrm{u}}$ in (b) has a small invariant mass we will call it a cluster and hadronize it by the procedure described in the text.

rection, see Fig. 2. This gives the system a small invariant mass, for which maybe only two-body final states are kinematically accessible. The continuum assumption above then is not valid, and the traditional iterative Lund scheme is not applicable. We call such a low-mass string a cluster, and treat it differently from above. When kinematically possible, a $\mathrm{c}-\overline{\mathrm{q}}$ cluster will decay into a charmed meson and a light meson by the production of a light quark-antiquark pair in the colour force field between the two cluster endpoints, with the new quark flavour selected according to the same rules as in normal string fragmentation. The decay kinematics is for the time being assumed to be isotropic in the rest frame of the cluster, which is the behaviour to be expected in the limit of vanishing phase space.

3. Cluster collapse. This is the extreme case of the above situation, where the string mass is so small that the cluster cannot decay into two hadrons. It is then assumed to collapse directly into a hadron resonance, inheriting the flavour content of the string endpoints. The original continuum of string/cluster masses is replaced by a discrete set of hadron masses, mainly $\mathrm{D}$ and $\mathrm{D}^{*}$. By local duality arguments [13] we assume that this does not change the total rate of charm production. This is related to the argument used in the $\mathrm{e}^{+} \mathrm{e}^{-} \rightarrow c \overline{\mathrm{c}}$ channel, that the cross section in the $\mathrm{J} / \psi$ and $\psi^{\prime}$ peaks is approximately equal to a purely perturbatively calculated $c \overline{\mathrm{c}}$ production cross section restricted to the below-D $\overline{\mathrm{D}}$-threshold region. Similar relations have also been studied e.g. for $\tau$ decay to hadrons [14], and there shown to be valid to good accuracy. In the current case, the presence of other strings in the event additionally allows soft-gluon exchanges to modify parton momenta as required to obtain correct hadron masses. Traditional factorization of short- and long-distance physics would then also protect the charm cross section. Local duality and factorization do not specify how to conserve the overall energy and momentum of an event, when a continuum of $\bar{c} d$ masses is to be replaced by a discrete $\mathrm{D}^{-}$one, however. This will therefore be one of the key points to be studied below.

Nature will not be so crude in its classification as we have been here, but hopefully our ansatz should be close enough to give a good first approximation.

Charm cross sections are often presented as functions of Feynman- $x, x_{\mathrm{F}}=p_{\mathrm{L}} / p_{\mathrm{L}, \max } \approx$ $2 p_{\mathrm{L}} / E_{\mathrm{CM}}$, i.e. the longitudinal momentum fraction of the meson in the rest frame of the event [2, 3, 4]. The $x_{\mathrm{F}}$ distribution for the different production channels in the standard Lund model is shown in Fig. 3, both for $\mathrm{D}^{-}$and $\mathrm{D}^{+}$. We use Pythia 6.1 [15 with standard parameters to generate the plots. 
(a) $D^{-}$production

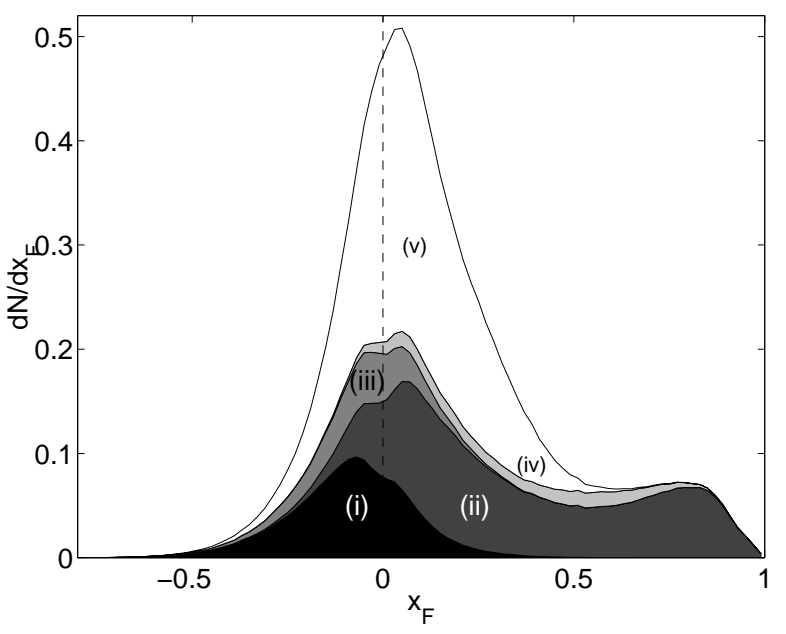

(b) $\mathrm{D}^{+}$production

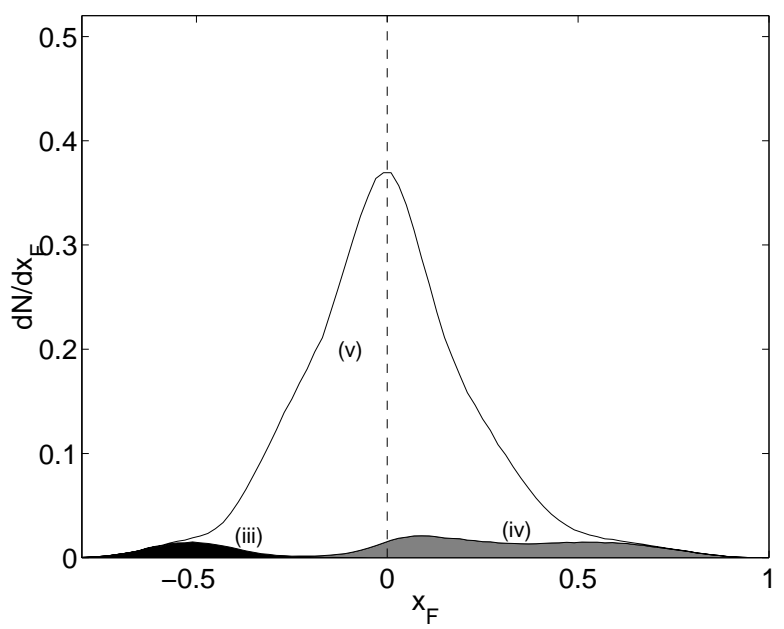

(c) Charm asymmetry

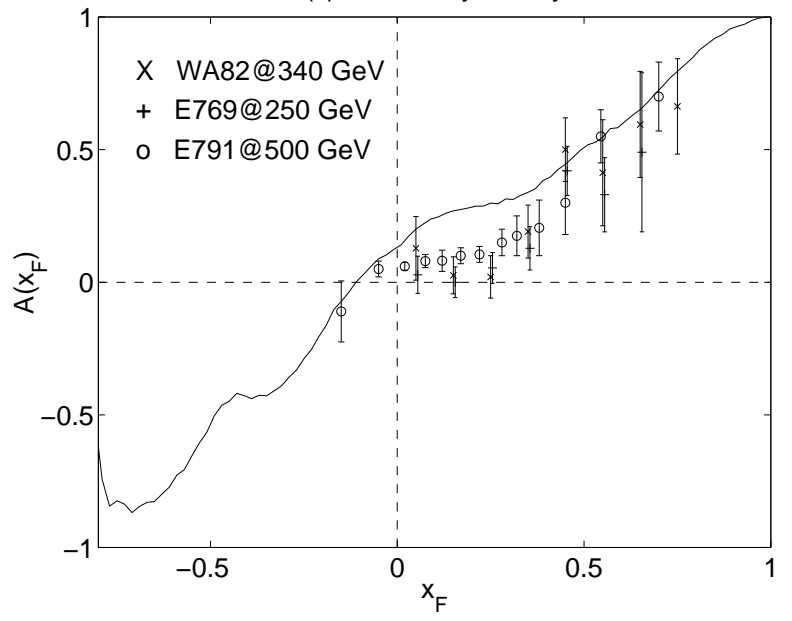

Figure 3: $\mathrm{D}^{\mp}$ meson production in a $\pi^{-} \mathrm{p}$ collision at a $\pi^{-}$beam momentum of $500 \mathrm{GeV}$,

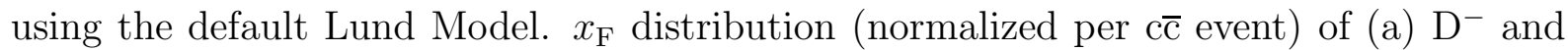
(b) $\mathrm{D}^{+}$for different production channels: (i) Cluster collapse, light quark from p end, (ii) Cluster collapse, light quark from $\pi^{-}$end, (iii) Cluster decay, light quark from p end, (iv) Cluster decay, light quark from $\pi^{-}$end and (v) String fragmentation. (c) The resulting asymmetry, Eq (11). Also shown is data from [2, 3, 4].

In perturbative QCD the $x_{\mathrm{F}}$ spectra of produced charm/anticharm quarks are identical to leading order, and the effects of higher orders are very small in this respect [5, 6]. Therefore any asymmetry between charm and anticharm hadrons is a simple measure of nonperturbative effects. In $\pi^{-} \mathrm{p}$ experiments the charm asymmetry is traditionally defined as

$$
A=A\left(x_{\mathrm{F}}, p_{\perp}\right)=\frac{\sigma\left(\mathrm{D}^{-}\right)-\sigma\left(\mathrm{D}^{+}\right)}{\sigma\left(\mathrm{D}^{-}\right)+\sigma\left(\mathrm{D}^{+}\right)},
$$

which measures the relative abundance of $\mathrm{D}^{-}$over $\mathrm{D}^{+}$as a function of $x_{\mathrm{F}}$ and $p_{\perp}$. We will here mainly consider the variation with $x_{\mathrm{F}}$, integrated over $p_{\perp}$, since $A$ does not vary much with $p_{\perp}$ over the experimental range of $x_{\mathrm{F}}$ values $[3$, 4. The asymmetry in PyтніA is compared to experiment in [2, 3, 4, and the model shows general agreement with experiment, except in the region $0 \leq x_{\mathrm{F}} \leq 0.4$ where the model is predicting a larger asymmetry than found in the data. One collaboration $\mathbb{4}$ has tuned the parameters of 
Pythia to obtain a good description of data also in this region, see below.

In our model the main source of asymmetry for all $x_{\mathrm{F}}>0$ is the production of $\mathrm{D}^{-}$ $(\bar{c} d)$ mesons via cluster collapse involving a valence $d$ from the pion beam, Fig. 3a. This production channel is not open for $\mathrm{D}^{+}(\mathrm{c} \overline{\mathrm{d}})$ since the $\pi^{-}$does not contain a valence $\overline{\mathrm{d}}$. A number of model aspects influence the rate and character of cluster collapses, and in the following we will review the main ones.

The charm mass enters in the perturbative matrix element as well as in the phase space, and so has a strong influence on the total charm cross section, but we have checked that the leading-order $x_{\mathrm{F}}$ distribution is not affected much. It also, together with the light-quark masses, sets the threshold of the charm-cluster mass spectrum. The c mass to use need not even be the same in the two applications, cf. the familiar distinction between current algebra and constituent masses. A large combined mass of the charm and light quarks in a cluster dramatically reduces the importance of the cluster collapse mechanism.

In the valence $u \bar{u}$ annihilation mechanism, the $\pi^{-}$beam remnant is a $\mathrm{d}$ quark, so no internal structure need be specified. In the gg fusion process, however, the remnant is a $\overline{\mathrm{u}} \mathrm{d}$ system in a colour octet state, i.e. attached to two strings. A convenient approach is to imagine this system split into two separate $\bar{u}$ and $d$ string endpoints. The beam remnant distribution function (BRDF) is introduced to describe how the (light-cone) momentum of the remnant is shared between the two, in fractions $\chi$ and $1-\chi$, respectively. For an octet meson remnant the $\chi$ distribution is always implicitly symmetrized between the $\mathrm{q}$ and $\overline{\mathrm{q}}$, while for an octet baryon remnant one quark (picked at random among the three) takes the fraction $\chi$ and the remaining colour antitriplet diquark $1-\chi$. To study the dependence of the asymmetry on the BRDF, we will consider two extreme cases and one intermediate. In one extreme one quark tends to take a small fraction of the available energy, much like the parton distributions in a hadron. In the other extreme, naive counting rules are used and the energy is, on the average, shared evenly between the quarks in the remnant. In Fig. 1 the individual spectra as well as the asymmetry is shown for the full $x_{\mathrm{F}}$ range.

It is interesting to note the difference between the regions $x_{\mathrm{F}}<0$ and $x_{\mathrm{F}}>0$. For $x_{\mathrm{F}}>0$ the $\mathrm{D}^{-}$is a leading particle and the asymmetry is attributed to cluster collapses involving a d-quark from the pion beam. In the proton fragmentation region $\left(x_{\mathrm{F}}<0\right)$ the $\mathrm{D}^{-}$is still leading and $\mathrm{D}^{+}$non-leading, so naively you would expect the asymmetry to be positive. However, when using an uneven sharing of energy in the proton beam remnant, diquark effects become prominent. As is seen in Fig. 2 the $\mathrm{c}$ is always connected to diquarks and the $\overline{\mathrm{c}}$ is connected to quarks. Therefore $\mathrm{D}^{+}$mesons produced from a cluster (containing a diquark) via cluster decay will, on the average, be harder than $\mathrm{D}^{-}$from cluster collapses, cf. Fig. 3. We see that there are two competing effects for $x_{\mathrm{F}}<0$, one that favours $\mathrm{D}^{+}$(diquark effect), and one that favours $\mathrm{D}^{-}$(cluster collapse). The strength of the diquark effect depends strongly on the assumed energy sharing in the proton beam remnant. This is seen clearly in Fig. Ec where an even sharing makes the asymmetry change sign for $x_{\mathrm{F}}<0$. If this region could be examined experimentally ( $\mathrm{p}$ beam on $\mathrm{p}$ target) it would give us a hint at which distribution should be used. Possibly one could use data from other (related) experiments to assess a reasonable choice of BRDF.

The partons entering the hard interaction are traditionally taken to have a nonvanishing primordial $k_{\perp}$, seen as a purely nonperturbative Fermi motion of partons inside the incoming hadrons, in addition to whatever is provided by perturbative gluon emission. Typical values should thus be 300-400 MeV. In many connections, also for charm in the current energy range [6], it has been noted that much higher values are required, at or 
(a) $D^{-}$production

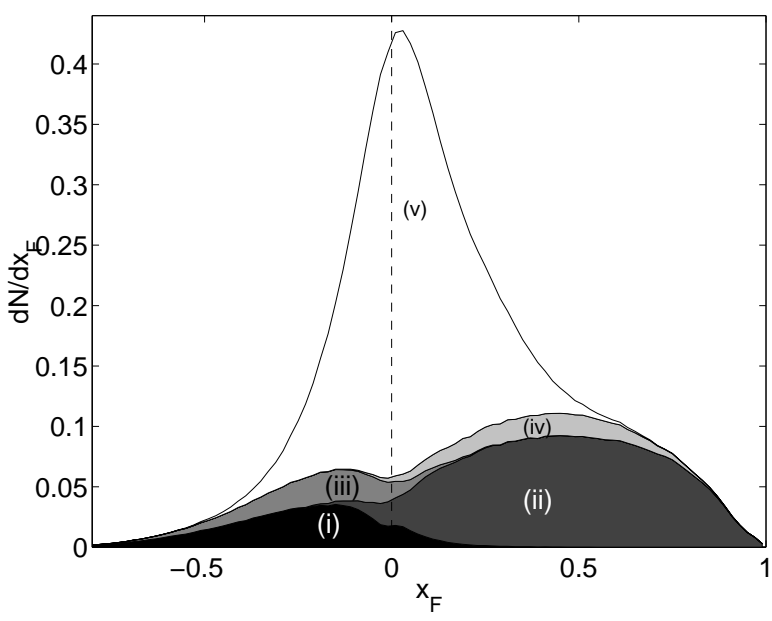

(b) $\mathrm{D}^{+}$production

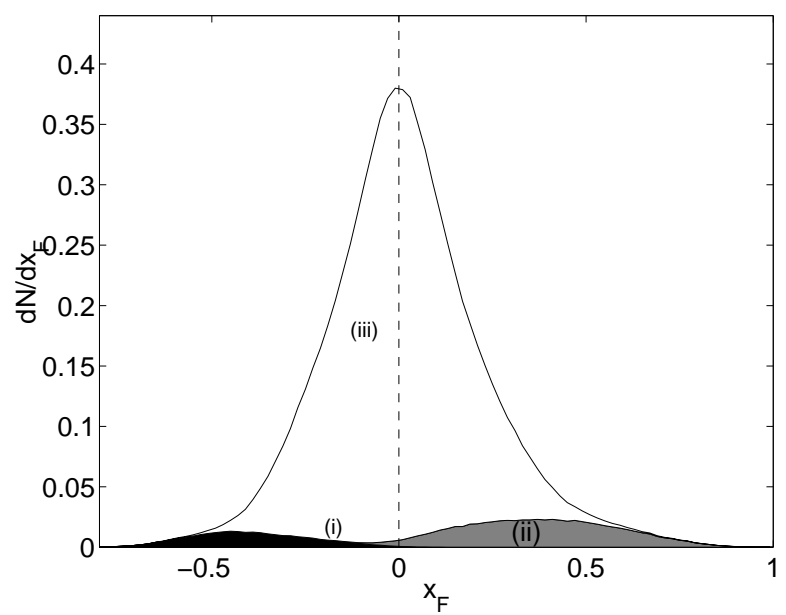

(c) Charm asymmetry

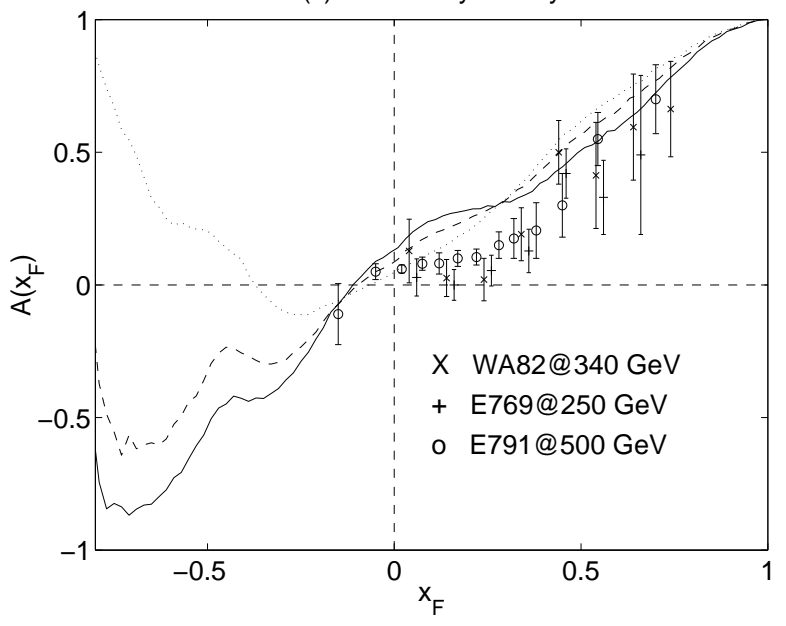

Figure 4: $x_{\mathrm{F}}$ distribution of (a) $\mathrm{D}^{-}$and (b) $\mathrm{D}^{+}$for different production channels (cf. Fig. 3, which corresponds to the default of extreme uneven sharing) in a $\pi^{-} p$ collision at a $\pi^{-}$beam momentum of $500 \mathrm{GeV}$ using an even sharing of energy between the quarks in a beam remnant. (c) the resulting asymmetry using three different choices of BRDF's: $\propto$ $(1-\chi)^{k} / \sqrt{\chi^{2}+c_{\min }^{2}}, \propto(1-\chi)^{k} / \sqrt[4]{\chi^{2}+c_{\min }^{2}}$ and $\propto(1-\chi)^{l}$ respectively, with $(k, l)=(1,0)$ for the pion and $(3,1)$ for the proton remnant, and $c_{\min }=0.6 \mathrm{GeV} / \mathrm{E}_{\mathrm{cm}}$. They correspond to an uneven (full), intermediate (dashed) and even (dotted) sharing of energy. For the $\pi^{-}$the three cases correspond to $\langle\chi\rangle=0.14,0.23$ and 0.5 respectively. Also shown is data from [2, 负, 四.

above $1 \mathrm{GeV}$. This remains somewhat of a mystery, which we do not attempt to solve here. The choice of primordial $k_{\perp}$ distribution is of non-negligible importance, both by providing a $p_{\perp}$ kick to the produced charm quarks and, by momentum conservation, an opposite kick to the beam remnants.

The cluster mass spectrum is affected both by the choice of charm mass, of BRDF's and of primordial $k_{\perp}$ distributions. As a typical example, the $\overline{\mathrm{c}}-\mathrm{d}$ singlet produced in Fig. 2a can have the mass distribution of Fig. 5la when calculated on the parton-level with $\mathrm{m}_{c}=1.35 \mathrm{GeV}$ and $\mathrm{m}_{d} \approx \mathrm{m}_{u} \approx 0$, or as in Fig. 5b if some parameters are varied. But we know that the observed mass spectrum of produced particles consists of peaks at the $\mathrm{D}^{-}$ and $\mathrm{D}^{*-}$ masses and then a continuum above the $\mathrm{D}^{-} \pi^{0}$ threshold, cf. Fig. 5a. 

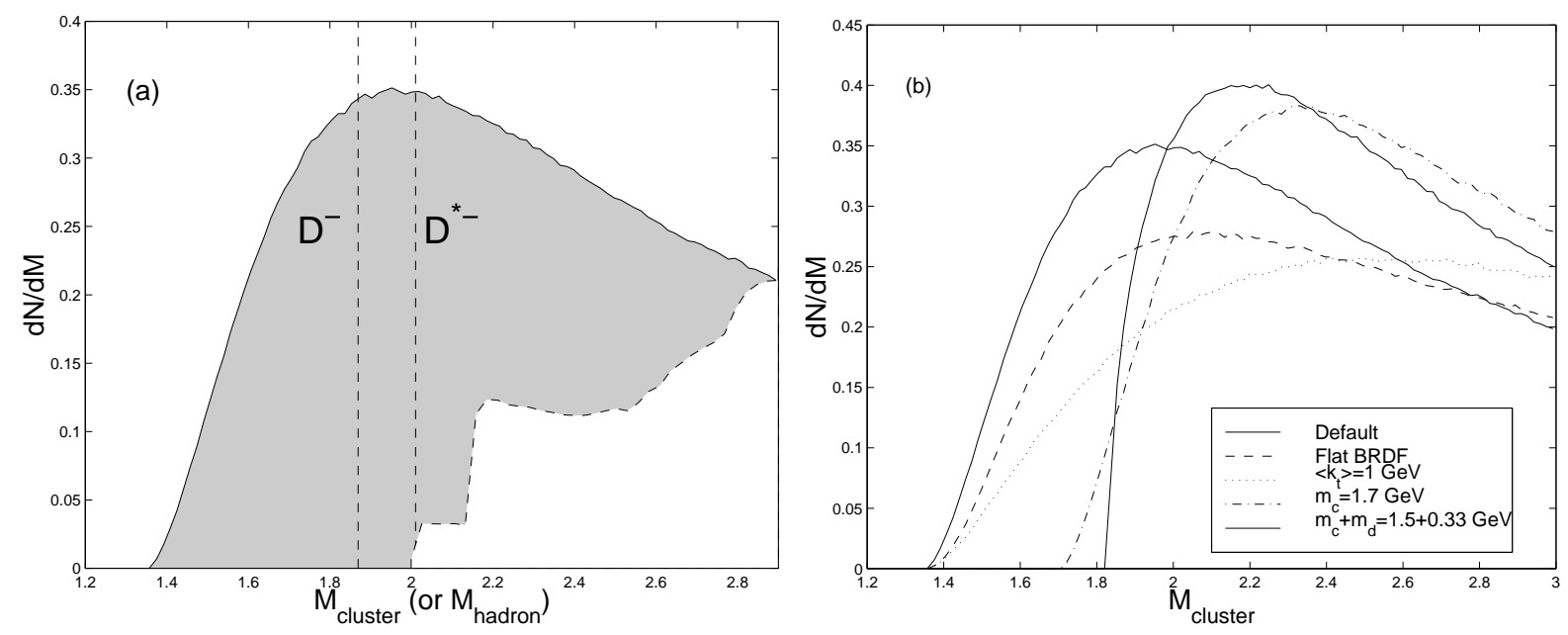

Figure 5: (a) Distribution of cluster (full) and meson (dashed) masses in the string model. Clusters within the gray area collapse to $\mathrm{D}^{-}$or $\mathrm{D}^{*-}$. (b) Dependence of the parton-level mass distribution on some parameters of the model.

The onset of the continuum depends on the assumed threshold behaviour. In one extreme, a $\mathrm{D} \pi$ pair is always formed when the cluster mass allows it. In another, this continuum state is only reached after a succession of thresholds: $\mathrm{D} \pi, \mathrm{D}^{*} \pi, \mathrm{D} \rho, \mathrm{D}^{*} \rho$, etc. Thus a fraction of events may collapse to a single resonance also above threshold, cf. the gray area in Fig. 5a. In the default implementation, exactly one attempt is made to form two hadrons by a random choice of allowed flavours and spins (using the string fragmentation relative probabilities), and is allowed to succeed if the sum of hadron masses is below the cluster mass. By increasing the number of attempts made before giving up, the behaviour interpolates to the first extreme above. Thus we see that the mass spectrum of collapsing clusters is affected in the low end by quark masses and in the high end by the transition to two-body states, altogether giving a large range of possible cluster collapse rates.

When a collapse occurs, confinement effects have to project the continuum of string masses onto the observed hadron mass spectrum. Because of the aforementioned local duality and factorization arguments, the total area of the spectrum should be conserved in the process. How the projection should be done is not known from first principles, however.

One conceivable strategy could be to introduce a weight function consisting of $\delta$ function peaks at the $\mathrm{D}^{-}$and $\mathrm{D}^{*-}$ masses, with suitably adjusted normalizations, and then a step function at the $\mathrm{D}^{-} \pi^{0}$ threshold. This weight function, when multiplied with the partonic mass spectrum, should then give the hadron-level mass spectrum. Such an approach is not well suited for Monte Carlo simulation, since the string mass is a complicated function of a number of variables and therefore the $\delta$ function cannot easily be integrated out. However, on general grounds, we do not expect the overall distribution of event characteristics to differ significantly between events with a $\bar{c}-\mathrm{d}$ string mass exactly equal to the $\mathrm{D}^{-}$one, and events where the string mass is maybe $100 \mathrm{MeV}$ off. An appealing shortcut therefore is to accept all partonic configurations and thereafter introduce some 'minimal' adjustments to the kinematics to allow hadrons to be produced on the mass shell. Such a strategy would be consistent not only with local duality arguments, but also with the presence of soft final-state interactions, i.e. the exchange of nonperturbative 
gluons that can carry some amount of momentum between the low-mass string and the surrounding hadronic system. In the following we will therefore adopt the language of 'gluons' transferring energy and momentum between the strings in a collision, while leaving unanswered the question on the exact nature of those 'gluons'. Specifically, we will not address the possibility of changes in the colour structure of events by such 'gluons'.

In this letter we will consider two different choices of energy shuffling schemes that can be said to be of opposite nature. We will further show that, as far as the asymmetry is concerned, the observable differences are small.

The first approach is the standard one in PYTHIA. It consists of shuffling momentum to the parton $i$ in the event that has the largest invariant mass when combined with the cluster $\mathrm{cl}$ that is supposed to collapse to a D (say). The new $i$ and D momenta are given by

$$
\begin{aligned}
p_{\mathrm{D}} & =(1-\delta) p_{\mathrm{cl}}+\epsilon p_{i}, \\
p_{i}^{\prime} & =(1-\epsilon) p_{i}+\delta p_{\mathrm{cl}}
\end{aligned}
$$

where $\epsilon$ and $\delta$ are determined by the conditions $p_{\mathrm{D}}^{2}=m_{\mathrm{D}}^{2}$ and $p_{i}^{\prime 2}=p_{i}^{2}=m_{i}^{2}$. Using the parton furthest away from the cluster has the advantage of minimizing the required momentum transfer, but does not offer a particularly appealing picture physically. In the following we therefore try to formulate a scheme where energy and momentum is shuffled to partons or strings in the vicinity of the cluster.

This problem can be approached in several ways, and we want to mention a few. The basic idea is to emit a soft gluon from the cluster, taking away the energy and momentum needed to put the remainder on the hadronic mass shell. One way to do this is simply to rescale the four-momentum of the cluster in order to give it the correct invariant mass. The gluon can then have both positive and negative masses and energies, which may be conceptually unappealing but not forbidden in principle. Worse is that such a rescaling can give $x_{\mathrm{F}}>1$, i.e. kinematical inconsistencies. Another approach is to let the cluster decay isotropically into a hadron and a massless gluon. The gluon can still have both positive and negative energies, but this is not a problem in itself since the gluon is connected to the nearest string piece, which is then hadronized according to the standard string-with-gluons scheme [16]. A gluon with positive energy will increase the energy and mass of the string while a gluon with negative energy will decrease it. It is the string which is the physical entity, and it of course has to have a positive energy and mass. The only observable effect of the extra gluon is a slight increase (decrease) in the average multiplicity in the phase space neighbourhood of the inserted positive (negative) energy gluon, but this effect is too small to be seen in an actual experiment. Even so, there are problems with the simple approach. For instance, if a negative-energy gluon is connected to a string that already has a small mass, it can give the string a negative mass and this is not permitted. So, if there are no sufficiently massive strings left in the event, the gluon cannot be connected anywhere, and this method cannot be used. Only if the radiated gluon has positive energy can the mechanism always be used without problems.

In practice, therefore, the negative-gluon approach is too error-prone. When the cluster mass is smaller than the intended hadron mass another approach is instead used to simulate the same effect, i.e. to take up energy from the vicinity of the cluster: a nearby string is allowed to emit a gluon which, when absorbed by the cluster, gives it the right mass. In more detail, imagine a string with endpoints $\mathrm{q}$ and $\overline{\mathrm{q}}$. Form a weighted sum of the endpoint momenta

$$
p_{s}=\alpha p_{\mathrm{q}}+\beta p_{\overline{\mathrm{q}}}=\frac{p_{\overline{\mathrm{q}}} p_{\mathrm{cl}}}{p_{\mathrm{q}} p_{\overline{\mathrm{q}}}} p_{\mathrm{q}}+\frac{p_{\mathrm{q}} p_{\mathrm{cl}}}{p_{\mathrm{q}} p_{\overline{\mathrm{q}}}} p_{\overline{\mathrm{q}}}
$$



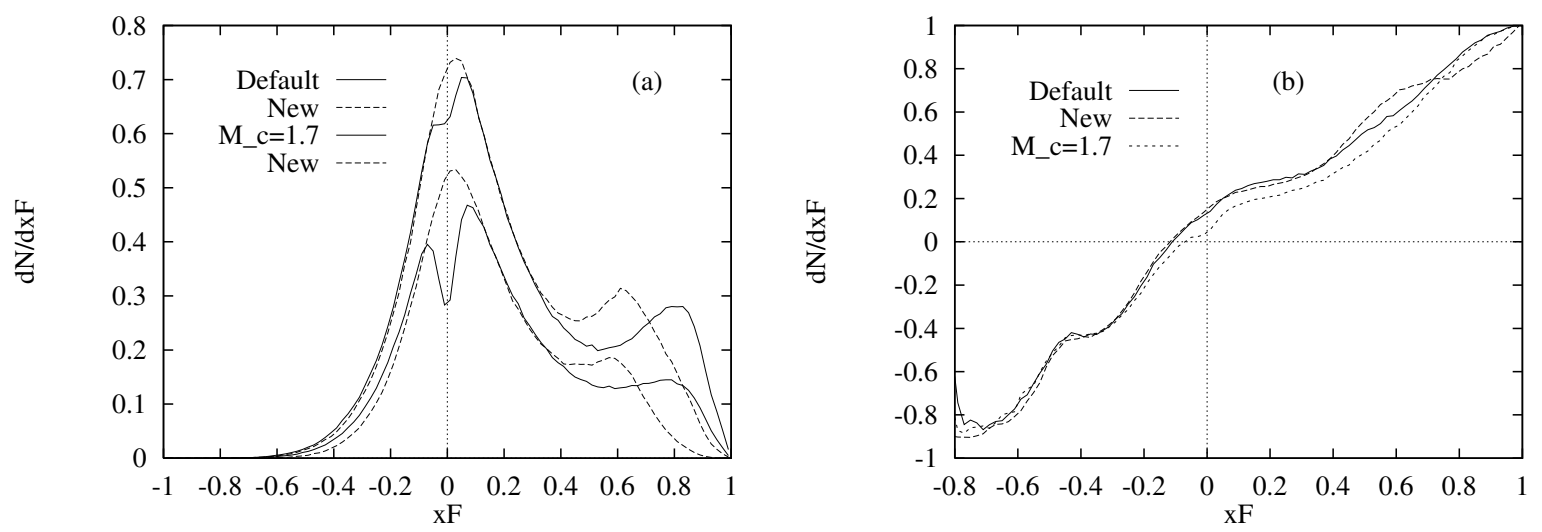

Figure 6: Some results for different cluster collapse algorithms: (a) The momentum spectrum for $\mathrm{D}^{-}$mesons produced from clusters (normalized to the total $\mathrm{D}^{-}$). The two top curves are with default parameters and the lower ones are with an increased charm mass (from 1.35 to $1.7 \mathrm{GeV}$ ). (b) the resulting asymmetry.

so that the end of the string that is closest to the cluster is weighted up relative to the one further away. Thereafter define

$$
p_{\mathrm{D}}=p_{\mathrm{cl}}+\delta p_{s},
$$

with $\delta$ determined by the constraint $p_{\mathrm{D}}^{2}=m_{\mathrm{D}}^{2}$. The meson will then have the correct mass and the string endpoint momenta are scaled down by factors $1-\delta \alpha$ and $1-\delta \beta$, respectively. Special cases need to be introduced to avoid e.g. $1-\delta \alpha<0$, but these affect only a small fraction of the events, and can be left aside here.

In summary, the new algorithm transfers a 'gluon' from the cluster to the nearest string if the original cluster mass is above the D meson mass, and transfers a 'gluon' the other way if the mass instead is below. Some results are shown in Fig. 6. As can be seen, the largest difference is in the distribution of the collapsed mesons at high $x_{\mathrm{F}}$, where the spectrum is somewhat softer in the new scheme than in the old one. Another difference is around $x_{\mathrm{F}}=0$, where the old algorithm tends to push the meson away from the middle, creating an unphysical dip in the distribution. This effect is seen more clearly in the distributions for a larger charm mass. The distribution with $m_{\mathrm{c}}=1.7 \mathrm{GeV}$ is also included to show the relative importance of the collapse mechanism on the asymmetry. Clearly the charm mass - by regulating the amount of collapses - provides a much larger uncertainty (except possibly at very large $x_{\mathrm{F}}$ ) than the choice of collapse scheme but, if nothing else, the new algorithm remedies some cosmetic problems of the old one. The new algorithm also changes some other characteristics of the event, e.g. the mean multiplicity is changed by $\approx 2 \%$, but this too is a small effect compared to the other uncertainties that we have mentioned.

The E791 collaboration has produced a tuned version of Pythia [4]. The specific parameters involved have already been discussed above, so we here only provide a brief summary:

- The charm quark mass is increased from 1.35 to $1.7 \mathrm{GeV}$. The fraction of cluster collapses is thereby reduced by about $35 \%$, which reduces the asymmetry considerably for $x_{\mathrm{F}}>0$, as demonstrated in Fig. 6. This large charm mass may appear unrealistic but it is also possible to reduce the number of cluster collapses by increasing the light-quark masses and/or increasing the probability for a cluster with 

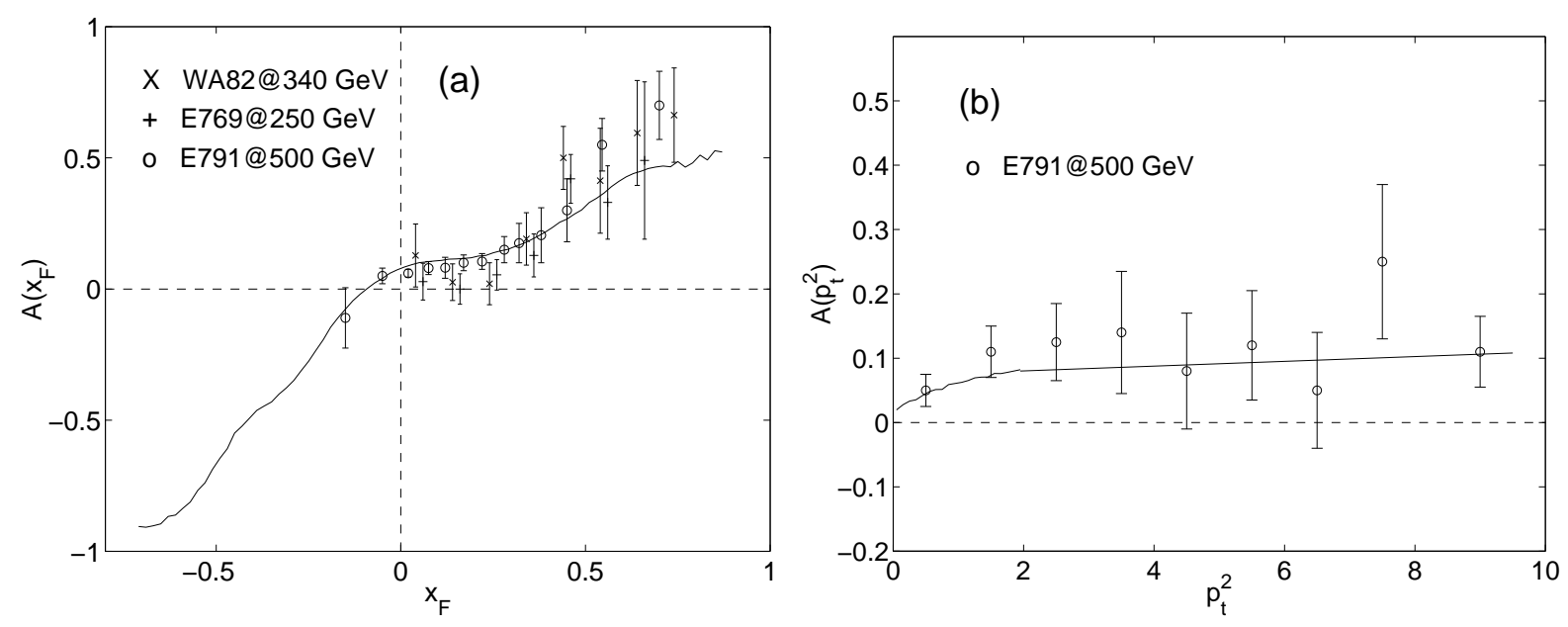

Figure 7: The $\mathrm{D}^{ \pm}$asymmetry as a function of (a) $x_{\mathrm{F}}$ and (b) $p_{\perp}^{2}$ for the modified version of Pyтнia described in the text: new cluster collapse mechanism, constituent masses for the quarks $\left(m_{c}=1.5 \mathrm{GeV}, m_{d}=m_{u}=0.33 \mathrm{GeV}\right)$, intermediate BRDF's, primordial $k_{\perp}=1.0$ and using two attempts to form two particles from a cluster. (a) is for all $p_{\perp}$ and (b) is for $-0.2 \leq x_{\mathrm{F}} \leq 0.8$. Experimental data is from [2, 3, 国.

a mass above the $\mathrm{D} \pi$ threshold to decay into two particles. By using constituent masses both for the light quarks and the charm quark (e.g. $m_{\mathrm{c}}=1.5 \mathrm{GeV}$ and $m_{\mathrm{d}}=m_{u}=0.33 \mathrm{GeV}$ ) and by allowing two attempts to form two hadrons from a cluster, the number of collapses is reduced by a comparable amount. A charm mass of $1.5 \mathrm{GeV}$ is also suggested from calculations of total cross sections for charm production in next-to-leading order QCD [6], and given the theoretical uncertainty regarding quark masses this value is not unreasonable.

- A somewhat less peaked BRDF, like our intermediate scenario. The asymmetry is reduced somewhat in the region $0<x_{\mathrm{F}}<0.4$, Fig. 1 . A photoproduction experiment [17] also seems to indicate flat BRDF's, but these are not determined by any basic principles, and are poorly known, so further studies will be needed here.

- The width of the Gaussian primordial $k_{\perp}$ distribution is increased from 0.44 to 1.0 $\mathrm{GeV}$. As we have noted above, such a number is unexpectedly large, but in agreement with other data, and therefore rather standard these days. This allows cluster collapses between a charm quark and a beam remnant to occur also at fairly large $p_{\perp}$ values, thus leading to an essentially $p_{\perp}$-independent asymmetry. Additionally, the $p_{\perp}$ kick added to charm quarks and beam remnants tend to increase the average invariant mass of the produced clusters, thereby reducing the number of cluster collapses.

Taken together, these parameter changes gives a good fit to data, both for the asymmetry and for the shape of the individual charm meson spectra. Fig. 7 shows the asymmetry using default PYTHIA with the following modifications: new cluster collapse mechanism, constituent quark masses, intermediate BRDF's, an increased intrinsic $k_{\perp}$ and with two attempts to form two hadrons from a cluster. The amount of cluster collapse is here reduced by about a factor of three compared with the default, to $\sim 16 \%$.

In summary, we have in this letter described the string fragmentation approach to charm production in hadronic collisions. A number of uncertainties have been identified and studied in detail, in particular the transition from a continuous string-mass distribu- 
tion to a discrete hadron-mass one. The conclusion is that the model can describe much of the existing data, but also that these data do not fully constrain the choice of model parameters. Further data on charm production in $\pi^{-} \mathrm{p}$ collisions may provide further information, as may charm production e.g. in ep collisions. Applications include, among other aspects, bottom production in hadron colliders where the asymmetry between $\mathrm{B}$ and $\overline{\mathrm{B}}$ mesons has to be understood in order to facilitate a study of $\mathrm{CP}$ violation effects [18. We intend to return to these and other related topics.

\section{References}

[1] M. Basile et al., Nuovo Cim. A66 (1981) 129;

ACCMOR Collaboration, R. Bailey et al., Phys. Lett. 132B (1983) 237;

NA27 Collaboration, M. Aguilar-Benitez et al., Phys. Lett. B161 (1985) 400;

NA32 Collaboration, S. Barlag et al., Z. Phys. C49 (1991) 555

[2] WA82 Collaboration, M. Adamovich et al., Phys. Lett. B305 (1993) 402

[3] E769 Collaboration, G.A. Alves et al., Phys. Rev. Lett. 72 (1994) 812

[4] E791 Collaboration, E.M. Aitala et al., Phys. Lett. B371 (1996) 157

[5] P. Nason, S.Dawson and R.K. Ellis, Nucl. Phys. D327 (1989) 49;

W. Beenakker, R. Meng, G.A. Schuler, J. Smith and W.L. Van Neerven, Nucl. Phys. B351 (1991) 507

[6] S. Frixione, M.L. Mangano, P. Nason and G. Ridolfi, Nucl. Phys. B431 (1994) 453

[7] Particle Data Group, C. Caso et al., Eur. Phys. J. C3 (1998) 1

[8] V. Barger, F. Halzen and W.Y. Keung, Phys. Rev. D25 (1982) 112;

P. Mazzanti and S. Wada, Phys. Rev. D26 (1982) 602;

G.I. Lykasov and M.N Sergeenko, Z. Phys. C56 (1992) 697;

R.C. Hwa, Phys. Rev. D51 (1995) 85;

V.A. Bednyakov, Mod. Phys. Lett. 68 (1995) 61;

A.K. Likhoded and S.R. Slabospitsky, hep-ph/9710476;

E. Cuautle, G. Herrera and J. Magnin, Eur. Phys. J. C2 (1998) 473;

O. Piskounova, Nucl. Phys. B50 (Proc. Suppl.) (1996) 179;

J. Dias de Deus and F.O. Durães, hep-ph/9803449

[9] B. Andersson, H.-U. Bengtsson and G. Gustafson, Lund preprint LU TP 83-4 (1983)

[10] S.J. Brodsky, P. Hoyer, C. Peterson and N. Sakai, Phys. Lett. 93B (1980) 451;

R. Vogt and S.J. Brodsky, Nucl. Phys. B478 (1996) 311;

G. Ingelman and M. Thunman, Z. Phys. C73 (1997) 505

[11] B. Andersson, G. Gustafson, G. Ingelman and T. Sjöstrand, Phys. Rep. 97 (1983) 31

[12] H.-U. Bengtsson, Comput. Phys. Commun. 31 (1984) 323 
[13] E.D. Bloom and F.J. Gilman, Phys. Rev. D4 (1971) 2901;

J.J. Sakurai, Phys. Lett. 46B (1973) 207;

H. Fritzsch, Phys. Lett. 67B (1977) 217;

R.A. Bertlmann, G. Launer and E. de Rafael, Nucl.Phys. B250 (1985) 61; and references therein.

[14] E. Braaten, S. Narison and A. Pich, Nucl. Phys. B373 (1992) 581

[15] T. Sjöstrand, Comput. Phys. Commun. 82 (1994) 74

[16] T. Sjöstrand, Nucl. Phys. B248 (1984) 469

[17] E687 Collaboration, P.L. Frabetti et al., Phys. Lett. B370 (1996) 222

[18] M. Chaichian and A. Fridman, Phys. Lett. B298 (1993) 218 